Images dans le monde ibérique et ibéricoaméricain

$8 \mid 2015$

L'Histoire et ses récits entre images, fictions et paratextes

\title{
El Gallardo español de Miguel de Cervantès : un théâtre aux frontières de l'histoire et de la poésie
}

Marie-Blanche Requejo Carrio

\section{(2) OpenEdition}

Journals

Édition électronique

URL : http://journals.openedition.org/agedor/388

DOI : 10.4000/agedor.388

ISSN : 2104-3353

Éditeur

Laboratoire LISAA

Référence électronique

Marie-Blanche Requejo Carrio, «El Gallardo español de Miguel de Cervantès : un théâtre aux frontières de l'histoire et de la poésie », L'Âge d'or [En ligne], 8 | 2015, mis en ligne le 01 février 2016, consulté le 21 avril 2019. URL : http://journals.openedition.org/agedor/388 ; DOI : 10.4000/agedor.388 


\title{
El Gallardo español de Miguel de Cervantès : un théâtre aux frontières de l'histoire et de la poésie
}

\begin{abstract}
Résumé : EL Gallardo español de Miguel de Cervantès est une comedia originale dans la production cervantine où le matériau historique est mis au service d'une fable romanesque. C'est à travers le jeu des burlas et des veras que nous explorons dans ce travail la fortune que connait l'opposition aristotélicienne entre Histoire et Poésie. Le brouillage des frontières qu'opère Cervantès permet de rendre compte d'une approche dynamique de la notion de vraisemblance qui voit se réconcilier l'histoire et le merveilleux. Nous explorons ainsi la singularité d'une écriture dramatique qui se démarque des théories aristotéliciennes et dont le moindre des paradoxes fut d'avoir connu plus de lecteurs que de spectateurs.
\end{abstract}

Mots-clés : comedia - Cervantès - comique - épique - vraisemblable - histoire - admiratio

Resumen: El Gallardo español de Miguel de Cervantes es una comedia singular en la producción cervantina en la que la materia histórica está al servicio de la fábula novelesca. El contraste entre las burlas y las veras nos permite rastrear en este estudio la fortuna que conoce la oposición aristotélica entre Historia y Poesía. La porosidad de las fronteras que observamos entre ambos polos nos permite dar cuenta de un concepto dinámico de la noción de verosimilitud que favorece el encuentro entre la Historia y lo maravilloso. Indagamos así la singularidad de una escritura dramática que se distingue de las teorías aristotélicas y cuya paradoja radica, entre otras cosas, en el hecho de haber conocido más lectores que espectadores.

Palabras clave: comedia - Cervantes - cómico - épico - verosimilitud - historia - dmiratio

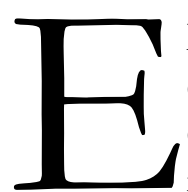

1 Gallardo español fait partie, avec Los baños de Argel, La gran sultana, La entretenida et Pedro de Urdemalas, des comedias de la dernière période de Cervantès. Sans entrer dans un débat d'érudits concernant la chronologie des pièces cervantines nous retiendrons avec Jean Canavaggio, que El Gallardo español a certainement été écrite entre 1606 et 1615, époque où Cervantès s’installe définitivement à Madrid ${ }^{1}$. On le sait, l'auteur du Quichotte s'adonne durant les dernières années de sa vie à une intense activité créatrice. Son œuvre romanesque triomphe, lui permettant de s'imposer enfin dans la république des Lettres. Mais il n'en délaisse pas moins le monde des planches et continue de cultiver une écriture dramatique qui l'a toujours passionné en dépit de ses échecs. L'on peut penser d'ailleurs que son succès de nouvelliste lui permet de reprendre avec assurance le métier de dramaturge, ne s'avouant pas vaincu face au triomphe de son éternel rival Lope de Vega. Les théories sur l'art dramatique qu'il expose dans ses écrits en témoignent: Don Quichotte (chap XLVIII, $1^{\text {re }}$ partie), El coloquio de los perros, l'Adjunta al Parnaso et bien sûr, son prologue au recueil dramatique qu'il publie en 1615, Ocho comedias y ocho entremeses nunca representados. Ne nous laissons pas abuser en effet par la demande de clémence qu'il adresse alors au lecteur pour son manque de modestie, Cervantès ne nous y a guère habitués ${ }^{2}$. Ne proclame-t-il pas dans son prologue aux Nouvelles exemplaires,

\footnotetext{
${ }^{1}$ CANAVAGGIO, Jean, Cervantès dramaturge, un théâtre à naître, Paris, PUF, 1977, p. 22.

${ }^{2}$ Dès l'orée du prologue, Cervantès avertit le lecteur : « No puedo dejar, lector carísimo, de suplicarte me perdones si vieres que en este prólogo salgo algún tanto de mi acostumbrada modestia », CERVANTĖS, Miguel de, Teatro
} 
publiées en 1613 : «Yo soy el primero que he novelado en lengua castellana »? Et deux ans plus tard, il n'hésite pas à revendiquer pour son théâtre un caractère tout aussi pionnier, même si cette fois il ne nous convainc pas aussi aisément : «Fui el primero que representase las imaginaciones y los pensamientos escondidos del alma, sacando figuras morales al teatro, con general y gustoso aplauso de los oyentes $»^{3}$. L'on peut bien sûr douter de cette assertion; il ressort néanmoins de ce prologue, cette conscience de l'écrivain d'occuper une place originale dans l'histoire du théâtre espagnol. Si Cervantès ne peut éviter de laisser transparaître une certaine amertume dans ses propos, car obligé de reconnaître les qualités de son rival prolifique, il n'en revendique pas moins les mérites de sa propre écriture, fidèle en cela à cette conscience d'artiste qu'il a toujours manifestée. Les propos que tient le chanoine du Quichotte peuvent être considérés comme un véritable manifeste de cette écriture dramatique qui ne saurait sacrifier au goût du temps. Évoquant l'attitude des directeurs des compagnies de théâtre dont le rôle était primordial dans la fortune d'un dramaturge, le personnage se fait l'écho de Cervantès en déclarant :

Acuérdome que un día dije a uno destos pertinaces : «Decidme, ¿no os acordáis que ha pocos años que se representaron en España tres tragedias que compuso un famoso poeta destos reinos, las cuales fueron tales que admiraron, alegraron y suspendieron a todos cuantos las oyeron, así simples como prudentes, así del vulgo como de los escogidos, y dieron más dineros a los representantes ellas tres solas que treinta de las mejores que después acá se han hecho ? »: "Sin duda - respondió el autor que digo - que debe de decir vuestra merced por La Isabela, La Filis y La Alejandra. » «Por esas digo - le repliqué yo -, y mirad si guardaban bien los preceptos del arte, y si por guardarlos dejaron de parecer lo que eran y de agradar a todo el mundo. Así que no está la falta en el vulgo, que pide disparates, sino en aquellos que no saben representar otra cosa. Sí, que no fue disparate La ingratitud vengada, ni le tuvo La Numancia, ni se le halló en la del Mercader amante, ni menos en La enemiga favorable, ni en otras algunas que de algunos entendidos poetas han sido compuestas, para fama y renombre suyo y para ganancia de los que las han representado $»^{4}$.

El Gallardo español est donc une œuvre de maturité, écrite à un moment où Cervantès est au faîte de son art. Sa présence dans le recueil de pièces dramatiques qu’il fait imprimer en 1614 est d'autant plus précieuse à nos yeux qu'elle témoigne par ailleurs d'une problématique consusbstantielle à son écriture, et plus particulièrement à son écriture dramatique, celle de l'opposition entre histoire et fiction. La comedia a ainsi semé la confusion parmi la critique entre ceux qui y ont vu une comedia a noticia, et ceux qui l'ont considérée comme comedia a fantasia ${ }^{5}$. D'aucuns ont vu dans l'œuvre l'exaltation d'un esprit nationaliste conformément à la geste épique qui la caractérise ${ }^{6}$, ou encore ont-ils mis l'accent sur sa structure mythique, sa valeur

completo (Florencio Sevilla Arroyo y Antonio Rey Hazas, eds.), Barcelona, ed. Planeta, 1987, p. 7. Toutes les citations du Gallardo español que nous proposerons dans le cours de cette étude seront issues de cette édition.

3 Ainsi que le rappellent Florencio Sevilla Arroyo et Antonio Rey Hazas dans leur édition, d'autres dramaturges avaient déjà eu recours à ces «figuras morales». En revanche, l’on peut reconnaitre le caractère pionnier de Cervantès pour ce qui est de l'extériorisation des sentiments de ses personnages. Ibid., note 14, p. 9.

${ }^{4}$ CERVANTÈS, Miguel de, Don Quijote de la Mancha (Francisco Rico, ed.), Barcelona, Crítica, 1998, I, chap. XLVIII, p. $552-553$.

${ }^{5}$ Considéré comme le premier traité de rhétorique de la Renaissance, la Propalladia de Torres Naharro distingue deux types de comedias, la «comedia a fantasía », de fiction pure et la "comedia a noticia », d'observation de la réalité : «Cuanto a los géneros de comedia, a mí me parece que bastarían dos para en nuestra lengua castellana : comedia a noticia y comedia a fantasía. A noticia se entiende de cosa nota y vista en realidad de verdad, como son Soldadesca y Tinellaria ; a fantasía, de cosa fantástica o fingida, que tenga color de verdad, aunque no lo sea, como son Serafina, Imenea, etc. », TORRES NAHARRO, Propalladia, in Federico SÁNCHEZ ESCRIBANO y Alberto PORQUERAS MAYO (dirs.), Preceptiva dramática española del Renacimiento y el Barroco, Madrid, Biblioteca Románica Hispánica, ed. Gredos, 1972, p. 64.

${ }^{6}$ CASALDUERO, Joaquín, Sentido y forma del teatro de Cervantes, Madrid, Gredos, 1966, p. 54-55.

${ }^{7}$ DE ARMAS, Frederick A., «Los excesos de Venus y Marte en El Gallardo Español», in Cervantes, su obra y su mundo : Actas del I Congreso Internacional sobre Cervantes (Manuel Criado de Val, ed.), Madrid, EDI-6, 1981, p. 249-259. 
romanesque $^{8}$, sur les différents jeux d'oppositions qui la caractérisent entre mythe, honneur et réalitée. D'autres en revanche ont mis l'accent sur ses résonances autobiographiques ou sa vraisemblance historique ${ }^{10}$.

Le Gallardo español, appartient en effet au cycle de comedias dites orientales ou encore turcobarbaresques. Avec los Tratos de Argel, los Baños de Argel et la Gran Sultana, le matériau historique qui la caractérise est emprunté à un passé récent voire, pour certaines de ces pièces, autobiographique. L'histoire racontée était très connue du grand public. Exemple d'héroïsme des troupes espagnoles, la pièce retrace un épisode notoire du siège d'Oran en 1563 par les turcs. La fidélité de Cervantès au déroulement des événements est remarquable ainsi que plusieurs études ont pu le faire apparaitree ${ }^{11}$. C'est à l'occasion d'un séjour à Oran en 1581 que Cervantès a certainement pu entendre des récits détaillés de la part des survivants du siège, dont le gouverneur de la place, don Martín de Córdoba. En outre, lors de son séjour à Alger, sans doute avait-il eu l'occasion d'échanger certaines informations avec un certain don Francisco de Valencia, à qui Philippe II avait confié la fortification des remparts de Mers-el-Kébir. À ces sources orales, s'ajoutent enfin les sources écrites puisque ce fait d'armes authentique est rapporté par Luis del Mármol Carvajal dans sa Descripción General de Affrica ${ }^{12}$. C'est cet épisode authentique de l'histoire de l'Espagne qui va permettre à Cervantès de tisser la trame épique d'une action sur laquelle vont se greffer les différents rebondissements d'une intrigue essentiellement romanesque. Nous ne nous attarderons pas sur la conformité d'un certain nombre d'éléments historiques que contient la comedia avec la chronique. Jean Canavaggio l'a magistralement démontré dans son étude sur le théâtre de Cervantès: l'assaut de Mérs-el-kébir et celui de la forteresse de San Salvador ${ }^{13}$, le rôle de don Martín de Córdoba, l'héroïsme de don Fernando de Cárcamo durant le siège, l'intervention des ingénieurs de l'armée espagnole dans la restauration des défenses de la ville, l'arrivée de la flotte de don Álvaro de Bazán, ainsi que les détails de la guerre de razzias dans cette frange de la frontière africaine sont autant de faits dont l'historicité est dûment attestée.

Objet de bien des controverses, le thème de l'Islam dans l'œuvre de Cervantès renvoie non seulement aux aléas de la politique impériale qui va marquer l'Espagne jusqu'aux premières décennies du XVII ${ }^{\mathrm{e}}$ siècle, mais il est surtout associé à une expérience personnelle au double visage : au fait d'armes glorieux se greffe l'expérience douloureuse de la captivité. El Gallardo español célèbre une action d'éclat de l'histoire de l'Espagne. En effet, l'exploit guerrier que retracent les aventures du vaillant Espagnol constitue le premier succès d'une politique méditerranéenne dont, quelques années plus tard, Lépante serait le couronnement: "la más memorable y alta ocasión que vieron los pasados siglos...» nous dit Cervantès dans le prologue des Nouvelles exemplaires ${ }^{14}$. Il convient toutefois de revenir, ne serait-ce que brièvement, sur la portée de ces victoires chrétiennes, à commencer par celle de Lépante. Si les Turcs subirent à cette occasion une cuisante défaite, ils n'en reconstituèrent pas moins pour autant leur flotte dans des délais assez brefs, et les défaites chrétiennes se succèdent ; la Goulette en 1574, Tunis peu de

\footnotetext{
8 ZIMIC, Stanislav, "Sobre la técnica dramática de Cervantes en El gallardo español», in Boletín de la Real Academia Española, 54, 1974, p. 505-18.

${ }^{9}$ Voir à ce sujet les études de William A. STAPP, «El Gallardo español. La fama como arbitrio de la realidad », in Cervantes, su obra y su mundo: Actas del I Congreso Internacional sobre Cervantes, op. cit., p. 261-272; Edward H. FRIEDMAN, The Unifying Concept: Approaches to the Structure of Cervantes' Comedias, York, S. C., Spanish Literature Publications Company, 1981, p. 29-30; Gethin HUGHES, "El gallardo español: a case of misplaced honour", Cervantes, 13, 1993, p. 65-75.

${ }^{10}$ CANAVAGGIO, Jean, Cervantès dramaturge, un théâtre à naître, op.cit., p. 53-56.

${ }^{11}$ Pour un état de la question concernant cet aspect de la comedia, nous renvoyons à l'étude de Jean CANAVAGGIO, ibid.

12 MÁRMOL CARVAJAL, Luis del, Descripción general de Affrica, Málaga, 1573, V, 28 : «Como Hascen Baxa fue sobre la ciudad de Oran y comabatio la villa de Marça el Quibir « (ff, 199v/206v). Cité par Jean Canavaggio, ibid. note 163, p. 88.

13 Appelée San Miguel par Cervantès, sans doute induit en erreur par Marmol lui-même comme le souligne J. CANAVAGGIO, ibid. p. 55.

${ }^{14}$ CERVANTES, Miguel de, Novelas ejemplares, ed. Jorge García López, Barcelona, Crítica, 2001, p. 17.
} 
temps après. Certes, l'on objectera avec Fernand Braudel que, malgré tout, c'est le mythe de l'invincibilité turque qui a été ébranlé, mais néanmoins l'Europe chrétienne n'a su profiter de son avantage et son déclin en Méditerranée a commencé. Or, c'est bien ce déclin qui se confirme quelques années plus tard au moment de la genèse du Gallardo español et que le siège d'Oran, en tant que symbole de la vie des présides, vient signifier. Sous Philippe III en effet, les tentatives de reprise d'une politique méditerranéenne échouent et, comme le rappellent Robert Ricard et Fernand Braudel, les places fortes du littoral africain, Le Peñón, Melilla, Gibraltar, sont progressivement abandonnées. Ces présides coûteux ont perdu leur importance stratégique réelle et incarnent davantage l'échec de projets ambitieux, comme aurait pu l'être la reprise de la politique méditerranéenne du cardinal Cisneros, que le souvenir d'un passé glorieux ${ }^{15}$. Ainsi donc, le présent dans lequel s'inscrit la genèse de la pièce projette sur la victoire espagnole un éclairage en demi-teinte. Au-delà d'un événement historique précis et circonscrit dans le temps, il nous invite à nous interroger plus largement sur son devenir. À l'heure où Cervantès écrit les exploits du Gallardo español, le temps n'est plus à l'héroïsme.

Étroitement lié dans son œuvre au thème de l'Islam, le thème de la captivité a fait l'objet de plusieurs projections littéraires, romanesques ou dramatiques, telles le récit du captif dans le Quichotte ou encore la comedia des Bagnes d'Alger. Ce thème revient sous la plume de Cervantès comme une obsession. María Antonia Garcés, il y a quelques années a réalisé sur ce sujet une étude fort éclairante où elle relit à la lumière d'une théorie psychanalytique du trauma, la captivité à Alger, l'esclavage, les menaces de mort, les tortures et autres scènes d'exécutions qu'elle considère comme des événements traumatisants pour lesquels l'écriture peut présenter des effets thérapeutiques $^{16}$. L'on peut garder ses distances par rapport à cette théorie, elle n'en a pas moins le mérite de proposer un regard différent sur une expérience que Cervantès n'a jamais pu évoquer qu'en la transposant dans la fiction.

Ces circonstances particulières nous semblent indispensables pour mieux comprendre ce rapport à l'histoire que le texte du Gallardo español met en œuvre, entre d'un côté ce que l'on pourrait appeler l'approche politico-idéologique, conforme à l'idéal hérö̈que que traverse tout récit épique et, de l'autre, la part autobiographique que suppose cette expérience de l'altérité qu'a signifiée la rencontre de Cervantès avec l'Islam. Le cadre historique dans lequel se déroule l'action sert en effet de toile de fond à la mise en place d'une double intrigue qui emprunte ses ressorts à toute une tradition littéraire ${ }^{17}$. Qu'il s'agisse du modèle de l'épopée classique - et du modèle troyen en particulier sur lequel repose le siège de la place - ou de la littérature chevaleresque et mauresque, la comedia multiplie les liens génériques afin de rendre vraisemblables les exploits de ses personnages et d'assurer le lecteur de la cohérence de leurs actions : Ulysse, Telamon, Hector, mais aussi Hercule, Roland, sont autant d'autorités convoquées comme gage de vraisemblance. Quant à la littérature mauresque, elle s'impose dès la première scène dans la conversation qui oppose les deux protagonistes maures, Arlaxa et Alimuzel, et permet de configurer cet espace si particulier de la frontière qui va autoriser l'amitié entre maures et chrétiens. Qu'il s'agisse de l'amitié entre Don Fernando et Alimuzel ou encore de la protection dont Arlaxa va entourer Doña Margarita, la conformité de la fable avec l'horizon d'attente ainsi défini dessine dès lors un premier niveau de vraisemblance de l'action représentée, favorisé par cette rencontre entre histoire et poésie.

Ce sont donc bien les liens de la comedia avec le récit épique que renforce ici l'idéal héroïque qui préside à la représentation des aventures du Gallardo español. À l'heure où Lope de Vega compose son grand poème épique La Jerusalén conquistada (1609), Cervantès, par ailleurs grand

\footnotetext{
15 Voir à ce sujet les chapitres que Fernand Braudel a consacrés à l'étude de ces pages de l'histoire de la Méditerranée. BRAUDEL, Fernand, La Méditerranée et le monde méditerranéen à l'époque de Philippe II, Paris, Armand Colin, 1990, II, p. 581-649.

16 ANTONIA GARCÉS, María, Cervantes en Argel, historia de un cautivo, Madrid, Gredos, 2005.

${ }^{17} \mathrm{Il}$ s'agit, rappelons-le, de la double intrigue qui naît de la rivalité amoureuse des soupirants d'Arlaxa, Alimuzel et Nacor, et des amours aventureuses de don Fernando de Saavedra et de Doña Margarita de Vaalderrama.
} 
admirateur comme on le sait du genre épique et d'Ercilla en particulier ${ }^{18}$, fait le choix, pour célébrer l'héroïsme de la nation, d'un genre dramatique que l'on pourrait qualifier d'hybride, car fait, souvenons-nous, pour être lu ${ }^{19}$. Et ce n'est pas là le moindre des paradoxes d'une comedia qui tisse étroitement les fils de la vérité historique et ceux de l'invention, conformément aux vœux de l'auteur énoncés dans les derniers vers de la comedia: "...llega el tiempo de dar fin a esta comedia cuyo principal intento ha sido mezclar verdades con fabulosos intentos $»^{20}$.

La familiarité de Cervantès avec les questions soulevées par les commentaires de la Poétique d'Aristote et reprises par les théoriciens de la Renaissance n'est plus à démontrer. L'auteur du Quichotte multiplie dans son œuvre, tant romanesque que dramatique, des commentaires théoriques qui montrent une connaissance approfondie des débats ayant nourri la réflexion des commentateurs d'Aristote. Le séjour en Italie (1569-1571) a en effet joué une part importante dans la formation intellectuelle de l'écrivain et de nombreux travaux ont mis au jour les traces laissées par la pensée du Tasse ou du Pinciano dans ses écrits ${ }^{21}$. Le rapprochement entre théâtre et livres de chevalerie que réalise à ce sujet le curé du Quichotte, en est l'exemple le plus connu. L'on y traite de l'opposition histoire-poésie, du vraisemblable, de la finalité de la poésie, du plaisir comme fonction principale de la comedia, de l'éthique qui doit être la sienne. Voici ce que nous dit le curé :

Y no sería bastante disculpa desto decir que el principal intento que las repúblicas bien ordenadas tienen permitiendo que se hagan públicas comedias es para entretener la comunidad con alguna honesta recreación y divertirla a veces de los malos humores que suele engendrar la ociosidad, y que pues este se consigue con cualquier comedia, buena o mala, no hay para qué poner leyes, no estrechar a los que las componen y representan a que las hagan como debían hacerse, pues, como he dicho, con cualquiera se consigue lo que con ellas se pretende. A lo cual respondería yo que este fin se conseguiría mucho mejor, sin comparación alguna, con las comedias buenas que con las no tales, porque de haber oído la comedia artificiosa y bien ordenada saldría el oyente alegre con las burlas, enseñado con las veras, admirado de los sucesos, discreto con las razones, advertido con los embustes, sagaz con los ejemplos, airado contra el vicio y enamorado de la virtud: que todos estos afectos ha de despertar la buena comedia en el ánimo del que la escuchare, por rústico y torpe que sea, y de toda imposibilidad es imposible dejar de alegrar y entretener, satisfacer y contentar la comedia que todas estas partes tuviere mucho más que aquella que careciere dellas, como por la mayor parte carecen estas que de ordinario agora se representan ${ }^{22}$.

Le principe de vraisemblance, enjeu principal de ces relations entre Histoire et Poésie, induit à un questionnement sans cesse renouvelé, sur la nature de la fiction et sa capacité à

\footnotetext{
${ }_{18} \mathrm{Au}$ cours de l'inventaire de la bibliothèque de don Quichotte, le curé déclare au sujet de La Araucana, d'Alonso de Ercilla, la Austriada de Juan Rufo et El Monserrato de Cristóbal de Virrués : «Todos esos tres libros [...] son los mejores que en verso heroico en lengua castellana están escritos, y pueden competir con los más famosos de Italia, guárdense como las más ricas prendas de poesía que tiene España ». CERVANTÈS, Miguel de, Don Quijote, op. cit, I, chap. VI, pp. 86-87. Mais déjà, dans le «Canto de Calíope » Ercilla avait fait l'objet d’un premier éloge : «Otro del mesmo nombre que de Arauco/ cantó las guerras y el valor de España/el cual los reinos donde habita Glauco/pasó y sintió la embravecida saña; no fue su voz, no fue su acento rauco, que uno y otro fue de gracia extraña, y tal, que Ercilla, en este hermoso asiento, merece eterno y sacro monumento. », CERVANTÈS, Miguel de, La Galatea (Francisco López Estrada y María Teresa López García-Berdoy, eds.), Madrid, Cátedra, 1995, p. 564.

${ }_{19}$ C'est là, nous semble-t-il, un des traits de ces comedias "jamais représentées », dont la facture si romanesque rappelle les antécédents de la comedia humaniste mais auxquelles se greffe un sens du spectacle propre à l'auteur du Quichotte. C'est avec un très grand intérêt que nous avons lu à ce sujet les pages que Daniel-Henri Pageaux a consacrées au théâtre de Cervantès, un «théâtre à lire », comme le suggère l'auteur. PAGEAUX, Daniel-Henri, Cervantes « raro inventor», de la poésie au roman, Paris, Librairie d'Amérique et d'Orient, 2011, p. 8 ss.

${ }^{20}$ CERVANTES, El Gallardo español, op. cit, vv. 3130-3134, p. 106.

${ }^{21}$ Nous renvoyons aux analyses désormais incontournables à ce sujet d'Edward O. RILEY, Teoría de la novela en Cervantes, Madrid, Taurus, 1981 et Alban K. FORCIONE, Cervantes, Aristotle and the Persiles, Princeton, Princeton University Press, 1970.

${ }^{22}$ CERVANTĖS, Miguel de, Don Quijote, op. cit, I, chap. XLVIII, p. 555.
} 
reproduire et à dépeindre le réel. La problématique, telle que Cervantès la pose dans el Gallardo español, illustre à notre avis, toutes les ambiguïtés et les paradoxes que la notion renferme ${ }^{23}$. À la Renaissance, en effet, les commentateurs d'Aristote se partagent entre ces deux pôles que sont vérité et mensonge. Entre ceux pour qui le vraisemblable doit se confondre avec l'histoire (Castelvetro, Piccolomini) et ceux dont l'interprétation du texte aristotélicien est plus nuancée, comme s'ils en avaient mieux perçu les ambiguités et pour qui la vraisemblance est une notion beaucoup dynamique qui ne se comprend qu'en fonction d'oxymores telles que le possible et l'impossible, le persuasif et l'incroyable, l'histoire et la poésie ${ }^{24}$.

Or, c'est bien à cette dynamique du «vrai-semblable » que les derniers vers de la comedia font allusion, reposant sur cette alliance de contraires qui prétend mêler les vérités aux fabuleuses inventions. Le contraste qui ressort de l'opposition verdades VS intentos, est une reformulation du traditionnel contraste burlas VS veras qui oppose le rire aux larmes, la plaisanterie à la civilité, le mensonge à la vérité ${ }^{25}$. Cervantès revendique de la sorte une contamination réciproque de la vie et de la littérature, de l'histoire et de la poésie, de la vérité et du mensonge qui se trouve au cœur de sa pratique littéraire. L'action du Gallardo español progresse dès lors à partir de tout un jeu d'oxymores qui brouille toute frontière entre les burlas et les veras. Et c'est dans cet espace frontalier singulier, que s'épanouit une fiction dramatique, certes nourrie par la tradition littéraire mais que franchit tantôt une écriture de l'histoire militaire de l'Espagne, tantôt les marques d'une instance référentielle singulière. Et ce sont bien ces incursions habilement menées qui confèrent à la fiction un aspect pseudo historique comme pour mieux la pervertir.

Dès l'acte I, une première occurrence du contraste burlas VS veras définit la nature des relations entre Arlaxa et Alimuzel lorsque la princesse, par caprice et curiosité, demande au chevalier transi de défier Don Fernando et de le lui amener prisonnier.

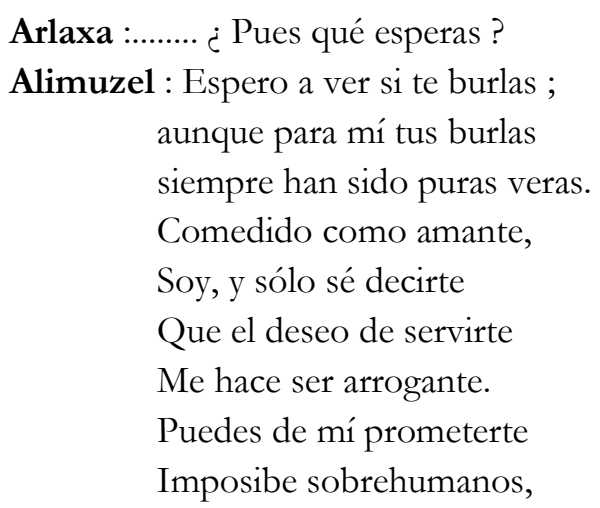

\footnotetext{
${ }^{23}$ C'est à partir de ce constat que, dans une étude sur le vraisemblable dans le Quichotte réfléchissant toute son acuité, Mercedes Blanco, distingue à partir de l'analyse des énoncés d'Aristote et du Pinciano, trois types de vraisemblable. Le premier, un vraisemblable philosophique où le poète sélectionne dans le possible ce qui est compatible avec le réel. En découle une action de type éthique ou psychologique exprimant ce qu'un homme ferait vraisemblablement ou nécessairement. Le deuxième, un vraisemblable rhétorique où l'action pour être persuasive doit refléter des idéaux collectifs. Ce vraisemblable est ce qui, à la lumière des préjugés, semble vrai. Enfin, le troisième type, un vraisemblable sophistique qui découle de la conception rhétorique : le réel peut devenir invraisemblable si le public n'y croit pas. Le faux et l'absurde peuvent dès lors devenir vraisemblables. Ce dernier type de vraisemblable admet l'irrationnel, le merveilleux, rejoint l'admiratio. BLANCO, Mercedes, «Vraisemblable et réel dans le Quichotte », in $L a$ Licorne, 39, 1996, p. 189-218.

${ }^{24}$ BLANCO, Mercedes, ibid., p. 201.

25 Dans un travail pionnier sur la bourle, Monique Joly a évoqué la productivité de ce contraste de la fin du XVe siècle à la deuxième moitié du XVII. Joly, Monique, La bourle et son interprétation. Recherches sur le passage de la facétie au roman (Espagne, XVI-XVII siècles), Toulouse, Université de Toulouse le Mirail, 1982 ; reimp. Atelier National de Reproduction des Thèses, Lille, 1986, p. 78.
} 
Mil prisioneros cristianos

Que vengan a obedecerte ${ }^{26}$.

Conformément au prototype du chevalier maure amoureux et larmoyant, Alimuzel se plaint de la rigueur de sa dame. Le vocable burlas qui permet de qualifier la requête incongrue d'Arlaxa, renvoie aux inventos du poète. C'est bien de ce caprice de princesse qui s'entiche d'un capitaine du camp adverse dont elle a entendu chanter les exploits, que démarre l'action dramatique ${ }^{27}$. Seuls la curiosité d'Arlaxa et son statut de dame rendent vraisemblable une action soumise à la logique implacable de la nécessité aristotélicienne : «le travail du poète ne consiste pas à dire ce qui s'est passé, mais bien ce qui pourrait se passer, les possibles, selon la vraisemblance ou la nécessité. $»^{28}$ Du haut de sa beauté et de sa supériorité, Arlaxa agit conformément aux raisons éthiques et psychologiques du modèle courtois qui l'inspire. C'est bien une causalité poétique qui régit l'enchaînement des actions et que rien ne devrait venir enrayer si ce n'est précisément la rencontre avec le singulier de l'histoire. Contrairement aux lois de la chevalerie, Don Fernando ne peut répondre sur-le-champ au défi que lui a lancé son rival. Les lois de la chevalerie s'opposent à la loi du soldat et à son devoir d'obéissance. À ce moment de l'intrigue, les veras d'Alimuzel se confondent avec le service de l'amant et renvoient à l'impossibilité de ramener vivant celui qu'Arlaxa dépeint comme un «Atlante de su España ${ }^{29}$ pour mieux souligner non seulement sa force surhumaine mais également l'exceptionnelle vaillance d'un soldat sur qui repose la destinée de son pays. Or, ces veras n'en vont pas moins rencontrer, sous les murailles d'Oran, les lois de la guerre : don Fernando est obligé de désobéir et de différer le duel. Mais dans le même temps Nacor, rival jaloux d'Alimuzel, le convainc qu'il s'agit là d'une ruse et le prince rejoint le douar de sa dame. L'honneur de don Fernando qui se présente au lieu du rendez-vous s'en trouve outrageusement bafoué. C'est alors que le vaillant soldat revêt l'habit des maures pour retrouver son adversaire retourné auprès des siens.

Ainsi donc, dès l'orée de l'intrigue, le contraste burlas VS veras, s'enrichit en permanence, non seulement de tout ce que la convention romanesque exige mais également de tout un ensemble de références à l'histoire contemporaine : rencontres et duels entre maures et chrétiens, vie des présides. Le travestissement de don Fernando en maure qui, tout au long de la pièce suscite l'incrédulité la plus totale et sème la confusion parmi les siens, témoigne de la façon dont la notion de vraisemblable fonctionne dans le texte. Nous reconnaissons d'une part le premier niveau d'une vraisemblance ontologique qui vient de la tension même que le vraisemblable entretient avec le réel. À cette vraisemblance ontologique, s'ajoute une vraisemblance poétique qui, au sein même de l'œuvre, garantit la logique d'une causalité de l'action représentée par sa conformité avec l'horizon d'attente qu'elle a contribué à mettre en place. Or, par le jeu des contrastes que Cervantès organise, cette vraisemblance intègre l'admiratio qui naît des multiples jeux de travestissements qui traversent l'œuvre: identité mauresque de don Fernando et, plus particulièrement, double métamorphose de doña Margarita en chevalier maure. La scène du duel entre Guzmán et don Fernando, multipliant les occurrences du verbe "ver », dévoile ainsi au spectateur le fonctionnement de l'illusion théâtrale et lui rappelle les effets illusionnistes d'un monde où rien n'est vrai ${ }^{30}$. Le jeu induit dès lors un troisième niveau de vraisemblance, rhétorique

${ }^{26}$ CERVANTĖS, Miguel de, El Gallardo Español, op. cit., vv. 49-60, p. 17.

${ }^{27} \mathrm{La}$ naissance de sentiments amoureux à partir du simple récit des exploits d'un valeureux guerrier est un thème traditionnel chez les novellieri de la Renaissance italienne. Nous le retrouvons entre autres, dans la nouvelle de l'Abencerraje, dans la version de l'Inventario qui interpole dans le récit des amours des deux héros maures, l'anecdote de la dame décidée à accorder ses faveurs à Rodrigo de Narváez après avoir entendu chanter ses exploits par son mari.

28 ARISTOTE, Poétique, 9, 1451a, in CEuvres (Richard Bodéüs, éd., Pierre Somville, trad.) Paris, Gallimard, 2014, p. 887.

${ }^{29}$ CERVANTES, El Gallardo español, op. cit, v. 41, p. 17.

30 À l'acte II, au cours de la razzia du douar d'Arlaxa par les chrétiens, Guzmán, capitaine chrétien, affronte don Fernando habillé en maure. A lieu alors le dialogue suivant : « G: ¡O eres diablo, o no eres hombre!/¿Quién te dio tal fuerza, perro? D. F.: No os admire ni os asombre,/ Guzmán, que haga este yerro/ quien respeta vuestro nombre. G: 
cette fois, qui permet à Cervantès de dénoncer de l'intérieur même de l'œuvre les mécanismes de l'illusion théâtrale. Si la productivité dramatique du contraste des burlas et des veras dans la comedia du Siècle d'Or n'est plus à démontrer ${ }^{31}$, leur ambivalence dans El Gallardo español requiert la lecture attentive du «lector carísimo », conformément aux déclarations du dramaturge lorsqu'il affirme dans Adjunta al Parnaso que s'il publie son théâtre c'est pour que le lecteur « vea de espacio lo que pasa apriesa y se disimula o no se entiende cuando las representan $»^{32}$

Cette conception dynamique d'un vraisemblable fondamentalement oxymorique, régit également les principes sur lesquels repose la construction du personnage de don Fernando de Saavedra. La tension entre honneur et devoir à laquelle le héros est soumis témoigne de cette contamination réciproque entre histoire et littérature. Toutes les actions de don Fernando sont en permanence vues à travers le prisme oblique de cette tension contradictoire. Objet d'une idéalisation poétique qui le hisse au niveau de la légende, l'hérö̈sme de don Fernando fait régulièrement l'objet de doutes. D'où l'ambiguïté d'un héroïsme qui s'exerce dans cet espace ambivalent où il est devenu bien difficile de discerner la frontière entre vérité historique et invention. L'onomastique elle-même est révélatrice de cette double filiation du personnage. Le modèle historique est don Francisco de Cárcamo. Quant à Saavedra, le patronyme du héros, il s'agit comme chacun sait non seulement de l'autre nom de Cervantès mais également du nom du captif du célèbre romance du "Río Verde » sur lequel se clôt la première partie de Las Guerras Civiles de Granada. $a^{33}$. Don Fernando est enfin, comme a pu le souligner la critique, ce vaillant espagnol, ce " gallardo español» de la Araucana qui réalise des exploits durant le siège de la forteresse de Tucapel ${ }^{34}$. C'est vers ces personnages aux visages protéiques, faits d'histoire et de fiction que convergent tous les regards de la comedia, tantôt incrédules tantôt aveugles de confiance, expression de la synthèse parfaite de ces burlas veras et de ces veras burlas.

Enfin, l'on reconnaîtra dans ce jeu des burlas VS veras du Gallardo español, destiné à brouiller les frontières entre vie et littérature, toute l'ironie qui caractérise l'écriture de l'auteur du Quichotte à chaque fois que le lecteur/ spectateur est invité à vérifier la véracité de ce qui est conté, autrement dit, vérifier la vérité du mensonge. C'est ainsi qu'au détour d'une didascalie, surgit une instance narrative à la première personne du singulier - marque qui nous rappelle le statut si particulier du théâtre cervantin, fait pour être lu - qui attire l'attention du lecteur sur le caractère véridique de ce qui est représenté. Entre en scène un soldat, Buitrago, demandant l'aumône pour les âmes du purgatoire (donner l'aumône pour les âmes du purgatoire, vouées à d'atroces souffrances était en effet considéré comme un acte d'extrême charité susceptible de procurer en retour sinon le salut de l'âme, du moins des avantages spirituels). Après avoir décrit le personnage, son entrée en scène et ses attributs, la didascalie précise : "Y esto de pedir para las ánimas es cuento verdadero, que yo lo vi, y la razón por que pedía se dice adelante. $»^{35}$

\footnotetext{
¿sois, a dicha, don Fernando? D. F.: El mismo que estáis mirando, aunque no me veis, amigo. G ¿Sois ya de Cristo enemigo? D.F. Ni de veras, ni burlando. », CERVANTÈS, Miguel de, El Gallardo español, op. cit., vv. 1742-1751, p. 66. ${ }^{31}$ Cf. JOLY, Monique, op.cit., p. 80 et note 34.

32 CERVANTÈS, Miguel de, Adjunta al Parnaso, Madrid (Vicente Gaos, ed.), Madrid, Clásicos Castalia, 1990 , p. 183.

${ }^{33}$ Ce romance «fronterizo » raconte la mort de Juan de Sayavedra et de Pedro Urdiales en 1448, tués au combat lors d'une escarmouche contre les maures dans le bassin du Río Verde. Outre l'intérêt que revêt l'homonymie du personnage avec le héros de Cervantès, nous remarquerons que le personnage historique de Juan de Sayavedra, contrairement à ce que chante le romance, n'est pas mort à l'occasion de cette bataille. Fait prisonnier, il finira par revenir à Séville à l'issue de sa captivité. Nous ne sommes pas en mesure de nous prononcer sur la connaissance que pouvait avoir Cervantès de ce mensonge de la Poésie à l'égard de l'Histoire auquel donne lieu le personnage. L'anecdote mérite toutefois d'être soulignée dans le cadre de cette étude. PÉREZ DE HITA, Ginés, Historia de los bandos Zegries y Abencerrajes (Primera parte de las guerras civiles de Granada) (Paula Blanchard-Demouge ed., Pedro Correa, estudio preliminar), Granada, ed Universidad de Granada, 1999, note 85, p. LXVI.

34 «No porque tantos vengan temor tiene/ el gallardo español ni esto le espanta,/ antes al escuadrón que espeso viene/ por mejor recibirle se adelanta. », ERCILLA, Alonso de, La Araucana (Isaías Lerner, ed.), Madrid, Cátedra, 1993 , p. 132.

35 C'est nous qui soulignons.
} 
La critique a abondamment commenté cette occurrence, y voyant un écho de la présence de Cervantès à Oran en qualité d'agent de Philippe $\mathrm{II}^{36}$. Ce « je » du narrateur qui revendique son statut de témoin oculaire pour mieux certifier l'authenticité des faits relatés n'est pas sans rappeler cette marque essentielle de l'écriture de l'histoire depuis Hérodote, qui fait de la première personne du singulier ou du pluriel la marque privilégiée du témoin, garant de la vérité des faits. L'originalité de cette occurrence, outre l'importance de son impact au niveau discursif dans une didascalie de théâtre, est son association au syntagme «cuento verdadero », syntagme construit sur un jeu d'opposition rappelant le statut mensonger de tout récit. D'où la nécessité d'un témoin qui en garantisse l'authenticité. La richesse sémantique du vocable cuento associé au qualificatif verdadero, nous amène à faire plusieurs observations.

Dans un premier temps, cette irruption $d u$ « je» du narrateur, dans une indication scénique où l'instance narratrice est traditionnellement neutre, fait office de captatio benevolentiae afin d'amener le lecteur à lire plus posément une scène dont la tonalité comique contraste avec le sujet évoqué $^{37}$ : la souffrance du soldat et sa faim. Outre les résonances avec les débats spirituels et religieux qui ont marqué l'époque ${ }^{38}$, le traitement comique auquel est soumise cette quête spirituelle, caractérise toute la distance ironique qu'établit Cervantès afin de montrer du doigt une réalité qu'il veut dénoncer. Buitrago, figure originale inspirée du miles gloriosus et du gracioso, soldat tiraillé par la faim et cherchant pitance par tous les moyens afin de mieux se battre, détourne en effet une quête au départ spirituelle à des fins personnelles : le produit de sa collecte doit lui permettre de remédier à sa souffrance. C'est bien la réalité de la vie misérable des présides, où le dénuement des soldats qui crèvent la faim est absolu, que le narrateur se charge de rappeler ainsi en qualité de témoin ${ }^{39}$. Or, comme de nombreuses études l'ont montré, la qualité de témoin dans l'œuvre de Cervantès ne suffit pas à garantir l'authenticité d'un fait. L'identité de ce dernier peut être douteuse et sa parole, dès lors, sujette à caution ${ }^{40}$. En effet, ce yo est le témoin oculaire privilégié d'événements dont le caractère récent peut être source de discorde avec le lecteur qui peut de son côté avoir parfaitement eu vent des événements et en contester la véracité. Cervantès anticipe-t-il ainsi les reproches que certains néo-aristotéliciens auraient pu lui formuler en choisissant de traiter d'un passé récent? Le Tasse dans son poème héroïque avait pourtant recommandé la représentation d'événements lointains pour éviter que le public, s'il avait vécu les événements en question, puisse en contester l'exactitude ${ }^{41}$. L'on se souviendra de l'épisode des faux-captifs du Persiles qui met en scène la supercherie de deux aigrefins, pris à leur propre mensonge ${ }^{42}$. Paradoxalement, cette proximité temporelle avec l'expérience du lecteur, garantit le sentiment d'une expérience partagée et place par là même ce dernier au cœur de l'illusion théâtrale pour mieux le toucher au moment où s'énonce une vérité fondamentale.

\footnotetext{
${ }^{36}$ Nous manquons hélas d'informations concernant l'activité et la mission de Cervantès à Oran à cette occasion. Mais Jean Canavaggio rappelle que cette mission a permis à Cervantès d'approcher certains survivants du siège dont D. Martín de Córdoba, gouverneur de la place. CANAVAGGIO, Jean, Cervantès dramaturge, un théâtre à naître, op. cit., p. 54.

37 Voir à ce sujet les propos de Cervantès cités précédemment dans Adjunta al Parnaso, op. cit., p. 183.

${ }^{38}$ En effet, cette aumône pour les âmes du purgatoire fait écho au climat religieux de l'époque et à des débats auxquels Cervantès, par le personnage de Buitrago, donne une réponse très personnelle, rappelant cette souffrance cruelle et élémentaire de l'homme sur terre, qu'est la faim. Cf., CERVANTĖS, Miguel de, El Gallardo español, op. cit., v. 628-656, pp. 34-36.

39 «La vie des présides ne pouvait être que misérable. Près de l'eau, les vivres pourrissent, les hommes meurent de fièvre. Le soldat crève de faim, à longueur d'année. » BRAUDEL, Fernand, La Méditerranée et le monde méditerranéen à l'époque de Philippe II, op. cit., t II, p. 611.

40 Voir à ce sujet la passionnante enquête menée par Michel Moner sur les stratégies des conteurs cervantins et l'ambivalence de leur parole. MONER, Michel, Cervantès conteur : écrits et paroles, Madrid, Casa de Velázquez, 1989.

${ }^{41}$ TASSO, Torquato, Discorsi dell'arte poetica e del poema eroico (Luigi Poma, ed.), Bari, Laterza, 1964.

${ }^{42}$ Nous nous permettons de renvoyer le lecteur à notre analyse de l'épisode où nous étudions ces jeux subtils de la vérité et du mensonge chers à Cervantès. REQUEJO, Marie-Blanche, « De cómo se guisa una fábula: el episodio de los falsos cautivos en El Persiles (III, X) », in Peregrinamente peregrinos, Actas del V Congreso Internacional de la Asociación de Cervantistas, Lisboa 1-5 septiembre 2003 (Alicia Villar Lecumberri, ed.), Asociación de Cervantistas, 2004, t.1, p. 861877 .
} 
Si nous considérons à présent le syntagme cuento verdadero, celui-ci est le lieu d'un oxymore qui renvoie aux manipulations dont le réel fait l'objet à partir du moment où il est mis en discours. Le syntagme rappelle que tout récit est suspect et qu'un certain nombre de garanties sont nécessaires pour qu'il puisse être considéré comme vrai. Tout témoin est un interprète de la réalité dont le discours est sujet à caution. L'instance de ce yo qui s'est exprimée à l'occasion de cette scène des âmes du purgatoire, soumet le texte à la présence invisible d'une forme d'autorité, qui se pose comme garante de l'authenticité d'un vrai monde qui existerait, lui, hors des frontières textuelles, les personnages de la fable prenant vie dans un univers fictionnel d'ores et déjà désigné comme «cuento » et donc comme discours mensonger. L'énoncé didascalique rejoint ainsi des problématiques chères à Cervantès, rappelant les termes du débat autour de la picaresque qui caractérise au niveau romanesque le dialogue de l'écrivain et d'Alemán. Au-delà du problème du statut générique de l'œuvre, l'occurrence de ce «je» brouille les frontières des genres et révèle l'ambition de Cervantès qui, en mêlant mensonge et vérité, mêle également le mode narratif et le mode dramatique, diégèse et mimèsis. El gallardo español nous apparaît dès lors comme le fruit de ces expérimentations littéraires auxquelles Cervantès se livre avec délectation dans sa recherche d'écriture d'une épopée comique ${ }^{43}$. L'expérimentation du Gallardo español, qui consiste à utiliser l'histoire pour rendre vrai-semblable une fantaisie romanesque comico-héroïque, pour mieux atteindre à cette "imitation parfaite » qu'il revendique dans le prologue du Quichotte, est ainsi l'occasion pour l'auteur de mettre à l'épreuve d'une création originale les préceptes aristotéliciens autour de la « vérité de l'histoire » en mettant dos à dos, le poète et l'historien : "Uno es escribir como poeta y otro como historiador $\gg{ }^{44}$.

Car n'est-ce-pas en effet du principe même d'autorité que se moque Cervantès dans le Gallardo español? Le poète commet dans cette pièce une singulière erreur : Alimuzel, le héros maure, meurt deux fois! Une première fois, à la fin de l'acte II, lors de l'attaque du douar d'Arlaxa et une deuxième fois à l'acte III, tué par don Fernando au moment du siège du fort de San Miguel. La première fois, le mort tombe sous les coups de Buitrago et en appelle à Allah :

Alimuzel : Muerto soy, Alá me ayude!

Arlaxa : ¡Acude Lozano, acude

Que han muerto a tu grande amigo!

(Cae Ali dentro, y éntrase Arlaxa tras él)

D. Fernando : Vengaréle en su enemigo,

Aunque de intención me mude ${ }^{45}$.

Il ressuscite quelques vers plus loin par la faveur d'Allah, ce même Dieu qu'Arlaxa évoque non sans quelque impatience, en réponse aux déclarations ferventes de son soupirant ressuscité :

(Entra Alimuzel)

Alimuzel : El remedio que aplicaste,

Bella Arlaxa, de tu mano,

Fue tal, que en él te mostraste

Ser un ángel soberano

Que a la vida me tornaste.

....

Arlaxa : Sosiégate y no me alabes,

Que el médico ha sido Alá

\footnotetext{
${ }^{43}$ BLANCO, Mercedes, «El Quijote y el Guðmán: dos políticas para la ficción », in Mateo Alemán y Miguel de Cervantes: dos genios marginales en el origen de la novela moderna (M. Guillemont y M. B. Requejo Carrió, eds.), Criticón, Presses Universitaires du Mirail, 101, 2007, p. 127-149.

${ }^{44}$ CERVANTÈS, Miguel de, Don Quijote, op. cit., t. II, chap. III, p. 649.

${ }^{45}$ CERVANTÈS, Miguel de, El gallardo español, op. cit., vv. 1807-1811, p. 68.
} 
De tus heridas tan graves ${ }^{46}$.

La seconde fois, Alimuzel est tué par D. Fernando dont il vient de découvrir la véritable identité : ¡Muerto me has, moro fingido/ y cristiano mal cristiano! ${ }^{47} \mathrm{Il}$ tombe derrière le rideau avant d'être secouru par don Fernando lui-même dans un ultime acte de bravoure absolue. Il réapparaittra peu de temps ensuite, entouré de pansements dans une dernière scène qui, enfin, le verra marié à Arlaxa. M. Gerli ${ }^{48}$ a rappelé comment Cervantès à cette occasion parodie le poème d'Homère et les deux morts de Priam qui constituent l'une des pièces centrales des polémiques de la renaissance autour du topos horacien «quandoque bonus dormitat homerus ». Le grand Homère, accusé d'erreur par Horace, est l'image de l'inattention du poète capable d'erreurs lorsqu'il se laisse entraîner par son imagination et ne respecte plus aucune limite à la vraisemblance. Or, cette même polémique est au cœur d'une discussion entre don Quichotte et Sansón Carrasco dans le chapitre III de la deuxième partie du Quichotte. Cervantès, par ce jeu prodigieux de la mise en abîme de sa propre écriture, évoque ses failles par ces mots :

Quisiera yo que los tales censuradores fueran más misericordiosos y menos escrupulosos, sin atenerse a los átomos del sol clarísimo de la obra de que murmuran: que si «aliquando bonus dormitat Homerus », consideren lo mucho que estuvo despierto por dar la luz de su obra con la menos sombra que pudiese, y quizá podría ser que lo que a ellos les parece mal fuesen lunares, que a las veces acrecientan la hermosura del rostro que los tiene: y, así, digo que es grandísimo el riesgo a que se pone el que imprime un libro, siendo de toda imposibilidad imposible componerle tal que satisfaga y contente a todos los que le leyeren ${ }^{49}$.

Dans El Gallardo español, la double mort d'Alimuzel est pour Cervantès l'occasion de convoquer Homère et Horace afin de mieux rappeler qu'il ne saurait y avoir d'œuvre parfaite et que la vraisemblance historique a ses limites. Les événements qui surviennent à la fin de la comedia ne font qu'ajouter à la confusion générale. C'est bien ce qu'exprime le personnage du comte que le récit de don Fernando a laissé encore plus perplexe : "Tan alegre destas cosas/estoy, cuanto estoy suspenso,/ porque dellas veo el fin y no imagino el comienzo. ${ }^{50}$ S'agit-il comme le suggère M. Gerli de l'échec de la synthèse des burlas et des veras? Nous sommes loin des positions de Lope de Vega pour qui, "todo puede ser uno, la Historia y la Poesía ${ }^{51}$. Pour Cervantès il semblerait, en effet que cet échec soit là pour rappeler le statut toujours problématique de la parole. La vérité de l'histoire est tout aussi sujette à caution que la vérité de la poésie. Nulle autorité n'est à l'abri d'une erreur. Tel est à nos yeux le sens de cet art poétique de la vérité et du mensonge dans cette comedia où, de manière paradoxale, Cervantès rappelle les limites de la vraisemblance historique comme pour mieux réconcilier, loin des préceptes aristotéliciens, l'histoire et le merveilleux.

El Gallardo español n'en finit pas de nous interroger. L'ambition déclarée de Cervantès à la fin de la pièce de mêler mensonge et vérité, à laquelle s'ajoutent les déclarations du comte qui n'a rien compris au récit des évènements qui lui a été fait, invite le lecteur à lire le texte à rebours

\footnotetext{
46 Ibid., v. 2043-2055, pp. 74-75.

${ }^{47}$ Ibid., vv. 2812-2813, p. 97.

${ }^{48}$ Voir à ce sujet l'article de Michael Gerli qui propose une très belle étude de la question d'un point de vue essentiellement épistémologique. GERLI, Michael E., «Aristotle in Africa: History, Fiction, and Truth in El gallardo español», in Cervantes, Bulletin of the Cervantes Society of America, 15, 2, 1995, p. 43-57.

${ }^{49}$ CERVANTÈS, Miguel de, Don Quijote, op. cit., t. II, chap. III, p. 654-655.

${ }^{50}$ CERVANTÈS, Miguel de, El Gallardo español, op. cit., vv. 3083-3085, p. 105.

51 Voir à ce sujet l'analyse que nous livre Charles V. AUBRUN, La comédie espagnole (1600-1680), Paris, PUF, 1966, p. 10 .
} 
confirmant ainsi le statut singulier d'une comedia faite pour être lue à défaut d'être représentée. Objet d'une interrogation originale des rapports entre Histoire et Poésie, El Gallardo español exploite la veine épique qu'offre un épisode glorieux de l'histoire de l'Espagne afin de poser les jalons d'une poétique héroïco-comique fondée sur le contraste des burlas VS veras ${ }^{52}$. Le vrai et le faux ne cessent de s'entrecroiser, obligeant le lecteur/ spectateur à s'interroger sur le rapport de la fiction dramatique à la vérité. La comedia prend ainsi les allures d'un véritable bal masqué où l'identité véritable des personnages principaux de l'œuvre, en particulier don Fernando et doña Margarita, fait l'objet d'un questionnement permanent. Cervantès, multipliant les jeux que favorise l'illusion dramatique, brouille à loisir les frontières entre le vrai et le faux afin de mieux nous amener à nous interroger non seulement sur la capacité du texte à réfléchir le réel, mais sur la façon dont la fiction elle-même est créatrice d'illusions.

Cette contamination réciproque entre histoire et littérature, gage de vraisemblance que préconise Aristote, autrement dit, le mode de mimèsis que met en œuvre Cervantès, rend compte d'une approche singulière du concept de vraisemblable. En dépit des déclarations théoriques en conformité avec les préceptes aristotéliciens qui émaillent ses écrits, la pratique que nous propose ici l'auteur du Quichotte est loin de se circonscrire à une simple concordance avec la pensée du Stagirite. En multipliant les points de contact entre histoire et poésie, Cervantès crée une zone frontière ambiguë où il appartient au lecteur de rechercher la vérité cachée. Au sein d'une œuvre qui ne cesse de s'interroger sur cette rupture fondamentale, El Gallardo español occupe à nos yeux une place de choix. Par ailleurs, la réponse qu'apporte ici l'écrivain en fait l'héritier d'une culture humaniste dont il conviendrait de repenser les contours, en particulier dans la relation de ce théâtre avec la comedia nueva de Lope de Vega.

\footnotetext{
52 LEWIS-SMITH, Paul, "Cervantes como poeta del heroísmo: de la Numancia a La gran sultana », in Memoria de la palabra, Actas del VI congreso de la asociación internacional del Siglo de Oro, Burgos-La Rioja 15-19 de julio 2002 (María Luisa Lobato, Francisco Domínguez Matito, eds.), Iberoamericana, Vervuert, 2004, II, p. 1155-1164.
} 